\title{
Spectral Element Analysis of the Transverse Vibration of a Plate
}

\author{
Ilwook Park, Minsik Lee, Usik Lee*, Oh-Yang Kwon \\ Department of Mechanical Engineering, Inha University, Inha-ro 100, Nam-ku, \\ Incheon 402-751, Republic of Korea \\ *Corresponding Author: ulee@ inha.ac.kr
}

\begin{abstract}
This paper presents a frequency-domain spectral element model for the rectangular finite plate element. The spectral element model is developed by using two methods in combination: (1) the boundary splitting; (2) the super spectral element method in which the Kantorovich method-based finite strip element method and the frequency-domain waveguide method are utilized.
\end{abstract}

Keywords: Plates, Spectral element method, Vibration.

\section{Introduction}

The finite element method (FEM) is one of most powerful computational methods to predict the dynamic responses or waves in a complex structure with arbitrary boundary conditions. As a high frequency excitation force can excite the higher vibration modes, the mesh sizes must be at least smaller than the wavelength of the highest vibration mode of interest in order to acquire sufficiently accurate dynamic responses at the high frequency. In such circumstances, the FEM will be computationally too expensive. Thus, the spectral element method (SEM), as a combination of the flexibility of the FEM and the accuracy of the continuum element methods, can be considered as an alternative solution technique.

In the literature, there are very few spectral element models for two-dimensional (2D) structures such as the plates and membranes which have finite dimensions in both $x$ and $y$ directions and being subjected to arbitrary boundary conditions. This is because, for such finite 2D structures, it is not easy to obtain exact free wave solutions in analytical forms which are required to formulate exact dynamic stiffness matrices or spectral element models.

In this paper, a new spectral element model proposed in the author's previous work ${ }^{(1)}$ for a finite rectangular plate element is introduced. The proposed spectral element model is formulated by using two methods in combination. The first one is the method of boundary splitting ${ }^{(2)}$ where the boundary conditions are split into several parts to form partial problems. The second method is the spectral super element method in which the Kantorovich method- based finite strip element method (in one direction) and the SEM (in the other direction) are used in combination. The performance of the proposed spectral element model is evaluated by the comparison with exact solutions and the solutions by the standard FEM.

\section{Theory}

The small amplitude transverse vibration of a thin plate can be represented by

$$
D\left(\frac{\partial^{4} w}{\partial x^{4}}+2 \frac{\partial^{4} w}{\partial x^{2} \partial y^{2}}+\frac{\partial^{4} w}{\partial y^{4}}\right)+\rho \frac{\partial^{2} w}{\partial t^{2}}=f(x, y, t)
$$

where $w(x, y, t)$ is the transverse displacement, $f(x, y, t)$ is the external force applied normal to the surface of the plate, $\rho$ is the mass per unit area of the plate, and $D$ is the flexural bending rigidity of the plate.

To formulate spectral element model for a thin plate by following the general procedure introduced in reference ${ }^{(4)}$, firstly all the time-domain quantities in the governing equations (1) are transformed into the frequency domain quantities by using the discrete Fourier transform (DFT) theory. For instance, the transverse displacement $w(x, y, t)$ and the external force $f(x, y, t)$ can be represented in the spectral forms as

$$
\begin{aligned}
\{w(x, y, t), & f(x, y, t)\} \\
& =\frac{1}{M} \sum_{m=0}^{M-1}\left\{\bar{w}_{m}(x, y), \bar{f}_{m}(x, y)\right\} e^{i \omega_{m} t}
\end{aligned}
$$


where $M$ is the number of samples for the fast Fourier transforms analysis and $\omega_{m}$ are the discrete frequencies up to the Nyquist frequency. In this paper, the over-barred quantities represent spectral components of the corresponding time domain quantifies. In the following derivation, the over-bars and the subscripts $m$ will be omitted for the sake of brevity.

The boundary splitting method used in this study and the original problem is represented by two partial problems: Problem A and Problem B. Problem A has the fixed boundary conditions on two parallel edges at $y=-L_{y} / 2$ and $L_{y} / 2$ and its solution is represented by $w_{A}(x, y)$. On the other hand, Problem B has the fixed boundary conditions on two parallel edges at $x=-L_{x} / 2$ and $L_{x} / 2$ and its solution is represented by $w_{B}(x, y)$.. The solution $w(x, y)$ to the original problem can be then obtained by summing the solutions to Problem A and Problem B.

For the derivations of $w_{A}(x, y)$ and $w_{B}(x, y)$ in the next sub-sections, the weak form of (8) can be obtained in the form as

$$
\begin{gathered}
\int_{x} \int_{y}\left\{D\left(\frac{\partial^{2} w}{\partial x^{2}}+v \frac{\partial^{2} w}{\partial y^{2}}\right) \delta\left(\frac{\partial^{2} w}{\partial x^{2}}\right)+2(1-v) D \frac{\partial^{2} w}{\partial x \partial y} \delta\left(\frac{\partial^{2} w}{\partial x \partial y}\right)\right. \\
\left.+D\left(\frac{\partial^{2} w}{\partial y^{2}}+v \frac{\partial^{2} w}{\partial x^{2}}\right) \delta\left(\frac{\partial^{2} w}{\partial y^{2}}\right)-\rho \omega^{2} w \delta w\right\} d x d y=0
\end{gathered}
$$

\subsection{Derivation of $w_{A}(x, y)$}

To obtain the solution $w_{A}(x, y)$ for the Problem A by using the SSEM, the rectangular finite plate element that has the dimensions $L_{x}$ and $L_{y}$ in the $x$ - and $y$-directions respectively is divided into a total of $N_{y}$ finite strip elements in the $y$-direction. The $e$ th finite strip element that has the width of $l_{y}^{(e)}$ in the $y$-direction. The displacement field $w_{A}(x, y)$ in the whole domain of the finite plate element can be then represented as

$$
w_{A}(x, y)=\boldsymbol{Y}_{A}(y) \boldsymbol{u}_{A}(x) \quad\left(-L_{y} / 2 \leq y \leq L_{y} / 2\right)
$$

By substituting (4) into (3), we can obtain an equation as

$$
\begin{aligned}
\boldsymbol{A}_{A 4} \frac{\partial^{4} \boldsymbol{u}_{A}(x)}{\partial x^{4}} & +\boldsymbol{A}_{A 2} \frac{\partial^{2} \boldsymbol{u}_{A}(x)}{\partial x^{2}} \\
& +\left(\boldsymbol{A}_{A 0}-\omega^{2} \boldsymbol{M}_{A}\right) \boldsymbol{u}_{A}(x)=0
\end{aligned}
$$

where $\boldsymbol{\Lambda}_{y 1}, \boldsymbol{\Lambda}_{y 2}, \boldsymbol{\Lambda}_{y 3}$, and $\boldsymbol{\Lambda}_{y 4}$ are constant matrices.
From Eq. (5), we can obtain an eigenvalue problem as

$$
\left[\boldsymbol{A}_{A 4} k_{x}^{4}+\boldsymbol{A}_{A 2} k_{x}^{2}+\boldsymbol{A}_{A 0}-\omega^{2} \boldsymbol{M}_{A}\right] \boldsymbol{r}_{A}=0
$$

By using the wavenumbers $k_{x(j)}$ obtained from (6), we can derive the solution to (5) in the form as

$$
w_{A}(x, y)=N_{A}(x, y ; \omega) d_{A}
$$

where $N_{A}$ is the dynamic shape function matrix for the Problem A.

\subsection{Derivation of $\mathrm{w}_{B}(\mathrm{x}, \mathrm{y})$}

The solution $w_{B}(x, y)$ of Problem B can be obtained from Problem A by simply rotating the coordinates $(x, y)$ $90^{\circ}$ degrees clock-wise. The only differences from Problem A are: (1) the fixed boundary conditions are placed at $x=-$ $L_{x} / 2$ and $L_{x} / 2$, and (2) the finite plate element is divided into $N_{x}$ finite strip elements in the $x$-direction. Thus, without repeating the similar procedure of solution derivation, the solution $w_{B}(x, y)$ for Problem $\mathrm{B}$ can be written as follows:

$$
w_{B}(x, y)=N_{B}(x, y ; \omega) d_{B}
$$

\subsection{Derivation of $\mathrm{w}_{\mathrm{B}}(\mathrm{x}, \mathrm{y})$}

The solution $w(x, y)$ for the original problem can be obtained by summing Eq. (7) and (8) as follows:

$$
\begin{aligned}
w(x, y) & =\boldsymbol{N}_{A}(x, y ; \omega) \boldsymbol{d}_{A}+\boldsymbol{N}_{B}(x, y ; \omega) \boldsymbol{d}_{B} \\
& =\boldsymbol{N}(x, y ; \omega) \boldsymbol{d}
\end{aligned}
$$

where $\boldsymbol{d}(\omega)$ is the $8 \times\left(N_{x}+N_{y}\right.$-2)-by-one spectral nodal DOFs vector.

\subsection{Formulation of Spectral Element}

The weak form of the governing equation of forced vibration can be derived as follows:

$$
\begin{aligned}
& \int_{x} \int_{y}\left[D\left(\frac{\partial^{2} w}{\partial x^{2}}+v \frac{\partial^{2} w}{\partial y^{2}}\right) \delta\left(\frac{\partial^{2} w}{\partial x^{2}}\right)+2(1-v) D \frac{\partial^{2} w}{\partial x \partial y} \delta\left(\frac{\partial^{2} w}{\partial x \partial y}\right)\right. \\
& +D\left(\frac{\partial^{2} w}{\partial y^{2}}+v \frac{\partial^{2} w}{\partial x^{2}}\right) \delta\left(\frac{\partial^{2} w}{\partial y^{2}}\right)-\rho \omega^{2} w \delta w d x d y \\
& =\int_{x} \int_{y} f(x, y) \delta w d x d y \\
& +\int_{y} V_{x 1} \delta w\left(-L_{x} / 2, y\right) d y+\int_{y} V_{x 2} \delta w\left(L_{x} / 2, y\right) d y \\
& +\int_{x} V_{y 1} \delta w\left(x,-L_{y} / 2\right) d x+\int_{x} V_{y 2} \delta w\left(x, L_{y} / 2\right) d x \\
& +\int_{y} M_{x 1} \delta\left(\partial w\left(-L_{x} / 2, y\right) / \partial x\right) d y+\int_{y} M_{x 2} \delta\left(\partial w\left(L_{x} / 2, y\right) / \partial x\right) d y \\
& +\int_{x} M_{y 1} \delta\left(\partial w\left(x,-L_{y} / 2\right) / \partial y\right) d x+\int_{x} M_{y 2} \delta\left(\partial w\left(x, L_{y} / 2\right) / \partial y\right) d x
\end{aligned}
$$


where $V_{x 1}(y), V_{x 2}(y), V_{y 1}(x)$, and $V_{y 2}(x)$ are the transverse shear forces applied on four boundary edges. Similarly $M_{x 1}(y), M_{x 2}(y), M_{y 1}(x)$, and $M_{y 2}(x)$ are the bending moments applied on four boundary edges.

By substituting (9) into (10), we can obtain the spectral element equation in the following form:

$$
S(\omega) d(\omega)=f_{1}(\omega)+f_{2}(\omega)
$$

\section{Numerical Results and Discussion}

To evaluate the performance of the present spectral element model, a simply supported uniform square plate as shown in Fig. 1(a) is considered. The present spectral element method (denoted by 'SEM') has been evaluated by comparing the natural frequencies obtained by the SEM with those obtained by the standard finite element method (denoted by 'FEM'). For the present example problem, exact solutions are available from the book by Meirovitch. ${ }^{(5)}$

Table 1 compares the natural frequencies of the simply supported square plate obtained by the present SEM with those by the standard FEM as well as with exact theory. For the SEM results, the square plate shown in Fig. 1(a) was represented by the one-element model as shown in Fig. 1(c) and the number of finite strip elements in the $x$ - and $y$-directions (i.e., $N_{x}$ and $N_{y}$ ) were increased until the natural frequency of the mode $(4,4)$ was sufficiently converged to exact solution. Similarly, for the FEM results, the number of finite elements used in the standard finite element analysis was also increased until the natural frequency of the mode $(4,4)$ was sufficiently converged to exact solution. Table 1 shows that the FEM results certainly converge to exact solutions when the total number of DOFs used in the analysis are increased up to about 43000 elements. Meanwhile, the SEM results are found to converge to exact solutions when the total number of DOFs employed in the one-element model is increased up to about 400 elements. This investigation shows that the same level of solution accuracy achieved by the standard FEM can be achieved by the present SEM by using an extremely small number of DOFs.

\section{Conclusions}

In this paper, the fundamental theory and concepts for the frequency-domain spectral element model proposed in the author's previous work ${ }^{(1)}$ for finite thin plates are briefly introduced. Through the example problem, extremely high accuracy and computational efficiency of the proposed SEM are verified by the comparison with the solutions by various solution techniques: exact solutions available in the literature and the standard FEM.

\section{Acknowledgment}

This research was supported by Basic Science Research Program through the National Research Foundation of Korea (NRF) funded by the Ministry of Science, ICT and Future Planning (Grant No: NRF2015R1A2A2A01003653)

Table 1. Comparison of the natural frequencies $(\mathrm{Hz})$ of a simply supported square plate obtained by the exact theory, $\mathrm{FEM}^{(6)}$, and $\mathrm{SEM}^{(1)}$

\begin{tabular}{|c|c|c|c|c|c|c|c|}
\hline \multirow{3}{*}{ Modes } & \multirow{3}{*}{ Exact } & \multicolumn{3}{|l|}{ FEM } & \multicolumn{3}{|l|}{ SEM } \\
\hline & & $n_{\mathrm{E}}=50 \times 50$ & $100 \times 100$ & $120 \times 120$ & $n_{\mathrm{E}}=1$ & 1 & 1 \\
\hline & & $n_{\mathrm{D}}=7399$ & 29799 & 42959 & $n_{\mathrm{D}}=72$ & 152 & 392 \\
\hline$(1,1)$ & 4.857 & 4.857 & 4.857 & 4.866 & 4.866 & 4.857 & 4.857 \\
\hline$(1,2)$ & 12.14 & 12.14 & 12.14 & 12.16 & 12.16 & 12.14 & 12.14 \\
\hline$(2,2)$ & 19.43 & 19.42 & 19.43 & 19.46 & 19.46 & 19.43 & 19.43 \\
\hline$(1,3)$ & 24.28 & 24.26 & 24.28 & 24.28 & 24.28 & 4.857 & 24.28 \\
\hline$(2,3)$ & 31.57 & 31.54 & 31.57 & 31.60 & 31.60 & 31.58 & 31.57 \\
\hline$(4,1)$ & 41.28 & 41.20 & 41.26 & 41.30 & 41.30 & 41.29 & 41.28 \\
\hline$(3,3)$ & 43.71 & 43.68 & 43.71 & 43.75 & 43.75 & 43.73 & 43.71 \\
\hline$(4,2)$ & 48.57 & 48.49 & 48.52 & 48.53 & 48.53 & 48.60 & 48.57 \\
\hline$(4,3)$ & 60.71 & 60.59 & 60.64 & 60.72 & 60.72 & 60.73 & 60.71 \\
\hline$(4,4)$ & 77.71 & 77.51 & 77.60 & 77.66 & 77.66 & 77.73 & 77.71 \\
\hline
\end{tabular}

Note: $n_{\mathrm{E}}=$ total number of finite elements; $n_{\mathrm{D}}=$ total number of degrees of freedom. 


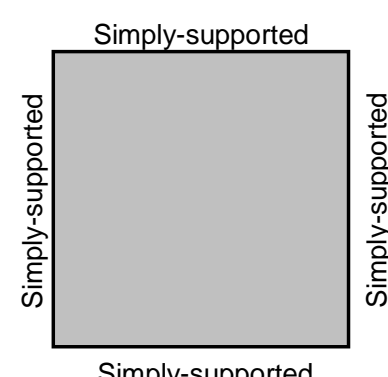

Simply-supported

(a) Example

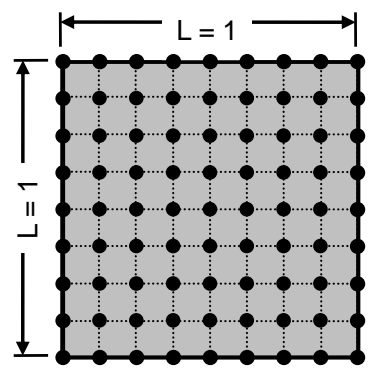

(b) FEM model

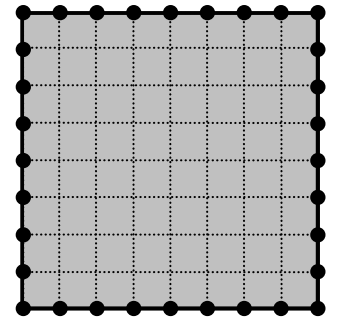

(c) SEM model

Fig. 1. An example: (a) Simply supported plate, (b) FEM model, and (c) SEM model. ${ }^{(1)}$

\section{References}

(1) I. Park, U. Lee, and D. Park: "Transverse vibration of the thin plates: Frequency-domain spectral element modeling and analysis", Mathematical Problems in Engineering, In Press, 2015.

(2) W. C. Reynolds: Solution of Partial Differential Equations, Lecture Note, Department of Mechanical Engineering, Stanford University, CA, USA, 1981.

(3) F. Birgersson, S. Finnveden, and C. M. Nilsson: "A spectral super element for modeling of plate vibration. Part 1: General theory", Journal of Sound and Vibration, vol. 287, No. 1-2, pp. 297-314, 2005.

(4) U. Lee: Spectral Element Method in Structural Dynamics, John Wiley \& Sons, Singapore, 2009.

(5) L. Meirovitch: Principles and Techniques of Vibrations, Prentice Hall, New Jersey, 1997.

(6) T. Y. Yang: Finite Element Structural Analysis, Prentice Hall, New Jersey, 1986 